\title{
Inflating branes inside abelian strings
}

\author{
Yves Brihaye* and Terence Delsate \\ Faculté des Sciences, Université de Mons-Hainaut, 7000 Mons, Belgium \\ Betti Hartmann ${ }^{\dagger}$ \\ School of Engineering and Science, \\ International University Bremen (IUB), 28725 Bremen, Germany
}

(Dated: July 31, 2018)

\begin{abstract}
We study a 6-dimensional brane world model with an abelian string residing in the two extra dimensions. We study both static as well as inflating branes and find analytic solutions for the case of trivial matter fields in the bulk. Next to singular space-times, we also find solutions which are regular including cigar-like universes as well as solutions with periodic metric functions. These latter solutions arise if in a singular space-time a static brane is replaced by an inflating brane. We determine the pattern of generic solutions for positive, negative and zero bulk cosmological constant.
\end{abstract}

PACS numbers: $04.20 . J b, 04.40 . \mathrm{Nr}, 04.50 .+\mathrm{h}, 11.10 . \mathrm{Kk}, 98.80 . \mathrm{Cq}$

\footnotetext{
*yves.brihaye@umh.ac.be

† b.hartmann@iu-bremen.de
} 


\section{INTRODUCTION}

The idea that we live in more than the observed 4 dimensions has been of huge interest ever since it was first suggested by Kaluza and Klein in the 1920s 1]. They studied a fivedimensional gravitational theory in a model with an extra compact dimension. The effective four dimensional theory then contains 4 dimensional gravity as well as the electromagnetic fields and a scalar field. In a similar way, some (super)string theories contain solutions in which extra dimensions (required for the consistency of those string theories) are all compact 2] (superstring theories have 6 extra compact dimensions). The size of these compact dimensions is of the order of the Planck length $=1.6 \cdot 10^{-33} \mathrm{~cm}$. An example is the Calabi-Yau space in heterotic string theory whose properties determine the low energy effective field theory.

Other models, which were discussed extensively in recent years are so-called brane world scenarios [3, 4, 15, 6, 7, 8, 9] which assume that the Standard model (SM) fields are confined to a 3-brane (a $3+1$ dimensional submanifold) which is embedded in a higher dimensional space-time. Some of the extra dimensions need now be non-compact.

Since gravity is a property of space-time itself, a model which describes appropriately the well-tested Newton's law should localise gravity well enough to the 3-brane. This was achieved in [8] by placing a 3-brane into 5 dimensions with the 5 th dimension being infinite. For the localisation of gravity in this model, the brane tension has to be fine-tuned to the negative bulk cosmological constant.

Recently, the localisation of gravity on different topological defects has been discussed 10]. This includes domain walls [11], Nielsen-Olesen strings [12, 13], and magnetic monopoles [14] in 5, 6 and 7 space-time dimensions, respectively. It was found [10, 12] that gravity-localising (so-called "warped") solutions are possible if certain relations between the defect's tensions hold. While in the case of domain walls and strings, gravity can only be localised when the bulk cosmological constant is negative, for magnetic monopoles the gravity-localisation is possible for both signs of the cosmological constant.

Brane world models including higher dimensional topological defects have recently also been discussed without bulk cosmological constant and from another point of view. 7dimensional brane worlds with a monopole (global and local) residing in the three extra

dimensions have been studied in [15]. The emphasis was put on the analysis of the locali- 
sation of the singularities in the 7-dimensional space-times. Inflating branes were studied, i.e. branes with a positive 4-dimensional cosmological constant and it was shown that the singularity can be removed from the space-time.

In this paper we study the model of [12, 13, 16, 17] for inflating branes. For completeness we also study the case of a positive bulk cosmological constant and determine the pattern of solutions.

Our paper is organised as follows : in section II, we present the 6 dimensional EinsteinAbelian-Higgs (EAH) model, we give the equations of motion and present special analytic solutions for trivial matter fields. In Section III, we present our numerical results. We give our conclusions in Section IV.

\section{THE MODEL}

We have the following 6-dimensional action [10]:

$$
S_{d}=S_{\text {gravity }}+S_{\text {brane }}
$$

where the standard gravity action reads

$$
S_{\text {gravity }}=-\int d^{6} x \sqrt{-g} \frac{1}{16 \pi G_{6}}\left(R+2 \hat{\Lambda}_{6}\right)
$$

$\hat{\Lambda}_{6}$ is the bulk cosmological constant, $G_{6}$ is the fundamental gravity scale with $G_{6}=1 / M_{p l(6)}^{4}$ and $g$ the determinant of the 6-dimensional metric.

The action $S_{\text {brane }}$ for the Einstein-Abelian-Higgs (EAH) string is given in analogy to the 4-dimensional case [18, 19] by:

$$
S_{\text {brane }}=\int d^{6} x \sqrt{-g_{6}}\left(-\frac{1}{4} F_{M N} F^{M N}+\frac{1}{2} D_{M} \phi D^{M} \phi^{*}-\frac{\lambda}{4}\left(\phi^{*} \phi-v^{2}\right)^{2}\right)
$$

with the covariant derivative $D_{M}=\nabla_{M}-i e A_{M}$ and the field strength $F_{M N}=\partial_{M} A_{N}-\partial_{N} A_{M}$ of the $\mathrm{U}(1)$ gauge potential $A_{M}$. $v$ is the vacuum expectation value of the complex valued Higgs field $\phi$ and $\lambda$ is the self-coupling constant of the Higgs field. 


\section{A. The Ansatz}

The Ansatz for the 6-dimensional metric reads:

$$
d s^{2}=M^{2}(\rho)\left[d t^{2}-\exp \left(2 \sqrt{\frac{\Lambda_{4}}{3}} t\right)\left(d r^{2}+r^{2} d \vartheta^{2}+r^{2} \sin ^{2} \vartheta d \varphi^{2}\right)\right]-d \rho^{2}-l^{2}(\rho) d \theta^{2},
$$

where $\rho$ and $\theta \in[0: 2 \pi]$ are the coordinates associated with the extra dimensions, while $r, \vartheta \in[0: \pi]$ and $\varphi \in[0: 2 \pi]$ are the 4-dimensional coordinates. $\Lambda_{4} \geq 0$ is the 4dimensional cosmological constant. The 4-dimensional metric satisfies the 4-dimensional Einstein equations:

$$
G_{\mu \nu}^{(4)}=8 \pi G_{4} \Lambda_{4} g_{\mu \nu}^{(4)} \quad, \quad \mu, \nu=0,1,2,3
$$

The space-time has four Killing vectors: $\frac{\partial}{\partial \theta}, \frac{\partial}{\partial \vartheta}, \frac{\partial}{\partial \varphi}$ and $\frac{\partial}{\partial t}$. Here we have replaced the 4-

dimensional flat Minkowski brane by an inflating brane whose 4-dimensional metric is given by the de Sitter metric. Note that we are using static coordinates here. The 4-dimensional curvature scalar associated with this inflating brane is then $R^{(4)}=4 \Lambda_{4}$. The motivation to use an inflating brane comes from the observationally confirmed assumption that out universe has a small positive cosmological constant.

For the gauge and Higgs field, we have [18]:

$$
\phi(\rho, \theta)=v f(\rho) e^{i n \theta}, \quad A_{\theta}(\rho, \theta)=\frac{1}{e}(n-P(\rho))
$$

where $n$ is the vorticity of the string, which throughout this paper we choose $n=1$.

\section{B. Equations of Motion}

Introducing the following dimensionless coordinate $x$ and the dimensionless function $L$ :

$$
x=\sqrt{\lambda} v \rho, \quad L(x)=\sqrt{\lambda} v l(\rho)
$$

the set of equations depends only on the following dimensionless coupling constants:

$$
\alpha=\frac{e^{2}}{\lambda}, \quad \gamma^{2}=8 \pi G_{6} v^{2}, \quad \Lambda=\frac{\hat{\Lambda}_{6}}{\lambda v^{2}}, \quad \beta^{2}=\frac{\hat{\beta}^{2}}{\lambda v^{4}}, \quad \kappa=\frac{8 \pi G_{4} \Lambda_{4}}{\lambda v^{2}}
$$

The gravitational equations then read :

$$
3 \frac{M^{\prime \prime}}{M}+\frac{L^{\prime \prime}}{L}+3 \frac{L^{\prime}}{L} \frac{M^{\prime}}{M}+3 \frac{M^{\prime 2}}{M^{2}}+\Lambda-\frac{\kappa}{M^{2}}=-\gamma^{2}\left(\frac{\left(f^{\prime}\right)^{2}}{2}+\frac{\left(1-f^{2}\right)^{2}}{4}+\frac{f^{2} P^{2}}{2 L^{2}}+\frac{P^{\prime 2}}{2 \alpha L^{2}}\right)
$$




$$
\begin{gathered}
6 \frac{M^{\prime 2}}{M^{2}}+4 \frac{L^{\prime}}{L} \frac{M^{\prime}}{M}+\Lambda-2 \frac{\kappa}{M^{2}}=-\gamma^{2}\left(-\frac{\left(f^{\prime}\right)^{2}}{2}+\frac{\left(1-f^{2}\right)^{2}}{4}+\frac{f^{2} P^{2}}{2 L^{2}}-\frac{P^{\prime 2}}{2 \alpha L^{2}}\right) \\
6 \frac{M^{\prime 2}}{M^{2}}+4 \frac{M^{\prime \prime}}{M}+\Lambda-2 \frac{\kappa}{M^{2}}=-\gamma^{2}\left(\frac{\left(f^{\prime}\right)^{2}}{2}+\frac{\left(1-f^{2}\right)^{2}}{4}-\frac{f^{2} P^{2}}{2 L^{2}}-\frac{P^{\prime 2}}{2 \alpha L^{2}}\right) .
\end{gathered}
$$

The Euler-Lagrange equations for the matter fields read:

$$
\frac{\left(M^{4} L f^{\prime}\right)^{\prime}}{M^{4} L}+\left(1-f^{2}\right) f-\frac{P^{2}}{L^{2}} f=0
$$

and

$$
\frac{L}{M^{4}}\left(\frac{M^{4} P^{\prime}}{L}\right)^{\prime}-\alpha f^{2} P=0
$$

The prime denotes the derivative with respect to $x$.

The equations (91)-(11) can be combined to obtain the following two differential equations for the two unknown metric functions:

$$
\frac{\left(M^{4} L^{\prime}\right)^{\prime}}{M^{4} L}+\frac{\Lambda}{2}=\frac{\gamma^{2}}{2}\left(\frac{P^{\prime 2}}{2 \alpha L^{2}}-\frac{1}{4}\left(1-f^{2}\right)^{2}\right)
$$

and

$$
\frac{\left(L M^{3} M^{\prime}\right)^{\prime}}{M^{4} L}+\frac{\Lambda}{2}-\frac{\kappa}{M^{2}}=-\frac{\gamma^{2}}{2}\left(\frac{2 P^{2} f^{2}}{L^{2}}+\frac{1}{4}\left(1-f^{2}\right)^{2}+\frac{1}{2} \frac{P^{\prime 2}}{\alpha L^{2}}\right)
$$

\section{4-dimensional effective action}

In the following we will discuss the 4-dimensional effective action arising in the limit $f(x) \equiv 1, P(x) \equiv 0$. The case where the matter fields are equal to their vacuum values is of interest to understand the asymptotic behaviour of the system in the generic case. Many publications have dealt with this, e.g. [20], since in contrast to the generic case analytic results are available.

Note that from (10) and (11) it follows that $L(x) \propto M^{\prime}(x)$ unless $M(x)$ is constant.

Due to the Ansatz we use for the metric, a dimensional reduction of the gravity action (2) can be performed. For this purpose notice that we can write the 6-dimensional Ricci scalar $R$ according to:

$$
R=\frac{R^{(4)}}{M^{2}}-8 \frac{M^{\prime \prime}}{M}-12 \frac{M^{2}}{M^{2}}-8 \frac{L^{\prime} M^{\prime}}{L M}-2 \frac{L^{\prime \prime}}{L}
$$


where $R^{(4)}$ is the 4-dimensional Ricci scalar associated with the metric on the brane. Using the fact that $L(x)=\tilde{c} M^{\prime}(x), \tilde{c}$ constant, we obtain the following 4-dimensional effective action:

$$
S_{\text {gravity }}=-\int d^{4} x \sqrt{-g^{(4)}} \frac{1}{16 \pi G_{\text {eff }}}\left(R+\Lambda_{\text {eff }}^{(4)}\right)
$$

where

$$
\Lambda_{e f f}^{(4)}=\left.\frac{2 \tilde{c} \pi G_{e f f}}{G_{6}}\left(\frac{2}{5} \hat{\Lambda}_{6} M^{5}-2 M^{\prime \prime} M^{4}-4 M^{3} M^{\prime 2}\right)\right|_{x=x_{1}} ^{x=x_{2}}
$$

and at first order, taking into account the fact that the matter fields are localized and will not influence the asymptotic form, we can define an effective coupling constant according to

$$
M_{p l}^{2}=\frac{1}{G_{e f f}}=\left.\frac{2 \tilde{c} \pi}{3 G_{6}} M^{5}\right|_{x=x_{1}} ^{x=x_{2}},
$$

where $x \in\left[x_{1}: x_{2}\right] . x_{2}-x_{1}$ is then the radius of the 2 -dimensional transverse space. The value of the parameter $\tilde{c}$ can be approximated by its value in absence of matter fields which can be evaluated exactly to be $\tilde{c}=4 /|2 \kappa-\Lambda|$.

\section{Special solutions}

Explicit solutions can be constructed for $f(x) \equiv 1$ and $P(x) \equiv 0$. These are by themselves of interest, of course, but are also interesting for the generic solutions since we would expect that the metric fields take the form presented below far away from the core of the string. A similar analysis has been done for global defects in [20].

\section{Static branes $\kappa=0$}

For $\Lambda=0$ the system admits two different types of solutions [16, 17]:

$$
M_{S}=1, L_{S}=x-x_{0}
$$

and

$$
M_{M}=\tilde{C}_{1}\left(x-\tilde{x_{0}}\right)^{2 / 5} \quad, \quad L_{M}=\tilde{C}_{2}\left(x-\tilde{x_{0}}\right)^{-3 / 5}
$$

where $\tilde{x}_{0}, \tilde{C}_{1}, \tilde{C}_{2}$ are arbitrary constants. By analogy to the 4-dimensional case, we refer to these solutions as to the "string" (S) and "Melvin" (M) branches, respectively. Note that the only difference to the 4-dimensional case are the powers in (21). 
For $\Lambda>0$ the explicit solutions read:

$$
M=C_{1} \sin \left(\lambda\left(x-x_{0}\right)\right)^{2 / 5} \quad, \quad L=C_{2} \frac{\cos \left(\lambda\left(x-x_{0}\right)\right)}{\sin \left(\lambda\left(x-x_{0}\right)\right)^{3 / 5}}
$$

where $C_{1}, C_{2}, x_{0}$ are arbitrary parameters and $\lambda^{2} \equiv 5 \Lambda / 8$. These solutions are periodic in the metric functions. In the 4-dimensional analogue, i.e. Nielsen-Olesen strings in de Sitter space, periodic solutions also appear for trivial matter fields [21, 22].

It is easy to see that the string and Melvin solutions (201), (21) can be obtained from these solutions for special choices of the free parameters and specific limits (for $\Lambda \rightarrow 0$ ) of the trigonometric solution.

The solutions for $\Lambda<0$ are given by

$$
M=A_{ \pm} \exp ( \pm \sigma x) \quad, \quad L=B_{ \pm} \exp ( \pm \sigma x) \quad, \quad \sigma^{2}=-\frac{\Lambda}{10}
$$

where $A_{ \pm}, B_{ \pm}$are arbitrary constants.

\section{Inflating branes $\kappa \neq 0$}

In the case $\kappa>0$ the solution for $M, L$ can be written in terms of quadratures as follows:

$$
x-\hat{x}_{0}=\int d M \sqrt{\frac{M^{3}}{\frac{\kappa}{3} M^{3}-\frac{\Lambda}{10} M^{5}+C}} \quad, \quad L=\tilde{L}_{0} \frac{d M}{d x}=\tilde{L}_{0} \sqrt{\frac{\frac{\kappa}{3} M^{3}-\frac{\Lambda}{10} M^{5}+C}{M^{3}}}
$$

where $\hat{x}_{0}, C$ are integration constants and $\tilde{L}_{0}$ is arbitrary. The explicit form of $M$ is involved. It depends on elliptic functions if $\Lambda=0$, in the case $\kappa=0$, the integration can be done by an elementary change of variable and leads to the expressions above. In the particular case $\Lambda=0$ and with $C=0$ we find

$$
M=\sqrt{\frac{\kappa}{3}}\left(x-\hat{x}_{0}\right) \quad, \quad L=L_{0} \equiv \tilde{L}_{0} \sqrt{\frac{\kappa}{3}}=\text { constant } .
$$

This latter solution corresponds to a cigar-type solution (in the extra dimensions) and we find that for $C \neq 0$ the solutions are also of this type, however cannot be given in an explicit form. "Cigar-type" refers to the fact that $g_{\theta \theta}=$ const.. Consequently, the circumference of a circle in the two extra dimensions becomes independent of the bulk radius $\rho$.

An analogue solution in 7 dimensions with monopoles residing in the three extra dimensions has been found previously in [15]. In the case $\Lambda>0, \kappa>0$ and $C=0$ we find

$$
M=\sqrt{\frac{10 \kappa}{3 \Lambda}} \sin \left(\sqrt{\frac{\Lambda}{10}}\left(x-\hat{x}_{0}\right)\right), \quad L=L_{0} \cos \left(\sqrt{\frac{\Lambda}{10}}\left(x-\hat{x}_{0}\right)\right) .
$$


Again, the metric functions are periodic. The periodicity of string-like solutions in de Sitter space seems to be a generic feature - independent of the number of space-time dimensions or of the type of brane present.

\section{E. Boundary conditions}

We require regularity at the origin $x=0$ which leads to the boundary conditions:

$$
f(0)=0, \quad P(0)=1, \quad M(0)=1,\left.\quad M^{\prime}\right|_{x=0}=0, \quad L(0)=0,\left.\quad L^{\prime}\right|_{x=0}=1 .
$$

Along with [12, 14, 15], we assume the matter fields to approach their usual asymptotic value far from the string core (i.e. for $r>>\sqrt{\lambda} v$ ) :

$$
f(x \rightarrow \infty)=1, \quad P(x \rightarrow \infty)=0 .
$$

\section{F. Asymptotic behaviour}

Close to the origin $x=0$, the functions have the following behaviour:

$$
\begin{aligned}
f(x<<1) & \simeq f_{0} x+f_{0}\left(\frac{1}{12} f_{0}^{2} \gamma+\frac{\gamma}{6 \alpha} p_{0}+\frac{1}{4} p_{0}+\frac{1}{48} \gamma+\frac{1}{12} \Lambda-\frac{1}{8}-\frac{1}{16} \kappa\right) x^{3} \\
P(x<<1) & \simeq 1+p_{0} x^{2} \\
M(x<<1) & \simeq 1+\left(\frac{1}{4} p_{0}^{2} \frac{\gamma}{\alpha}-\frac{1}{32} \gamma-\frac{1}{8} \Lambda+\frac{3}{16} \kappa\right) x^{2} \\
L(x<<1) & \simeq x+\left(-\frac{1}{6} f_{0}^{2} \gamma-\frac{5}{6} p_{0}^{2} \frac{\gamma}{\alpha}+\frac{1}{48} \gamma+\frac{1}{12} \Lambda-\frac{1}{4} \kappa\right) x^{3}
\end{aligned}
$$

For $\kappa=0$ the behaviour found in [12] is recovered.

For $x>>1$, the matter functions tend to their asymptotic values $P(x>>1) \rightarrow 0$, $f(x>>1) \rightarrow 1$. The metric functions thus tend asymptotically to the special solutions described above.

\section{NUMERICAL RESULTS}

Following the investigations in [15], we here mainly aim at a classification of the generic solutions available in the system. 


\section{A. Static branes $(\kappa=0)$}

We solve the system of ordinary differential equations subject to the above boundary conditions numerically. The system depends on three independent coupling constants $\alpha, \gamma$, $\Lambda$. We here fix $\alpha=2$, corresponding to the self dual case in flat space, and we will in the following describe the pattern of solutions in the $\gamma-\Lambda$ plane. Results for the 4-dimensional gravitating string [19] make us believe that the pattern of solutions for $\alpha \neq 2$ is qualitatively similar. For vorticity $n>1$, the situation might change (see 19]), however, we don't discuss this case here. As will become evident, the presence of two cosmological constants leads to a rather complicated pattern of solutions. The different types of solutions available are summarized is the Summary section. Their interconnection is illustrated in Fig 11.

\section{Zero or negative bulk cosmological constant}

The case $\Lambda=0$ was studied in detail in [16, 17]. We review the main results here to fit it into the overall pattern of solutions. The pattern of solution in the $\gamma$ - $\Lambda$-plane is given in Fig[1]

For $\Lambda=0$ and $\gamma<2$ two branches of solutions exist, with an asymptotic behaviour of the metric functions given by (201) and (21). Referring to their counterparts in a fourdimensional space-time [19] we denote these two families of solutions as the "string" and "Melvin" branches, respectively. The terminology used e.g. in [19] will be used throughout the rest of the paper.

Specifically for $\alpha=2$, we have $M(x) \equiv 1$ and $L(x>>1) \sim a x+b, a>0$. At $\gamma=2$ the two solutions coincide and $L(x>>1)=1$. When the parameter $\gamma$ is increased to values larger than 2 the string and Melvin solutions get progressively deformed into closed solutions with zeros of the metric functions. For $\gamma>2$ indeed, the the string branch smoothly evolves into the so called inverted string branch (again using the terminology of [19]). The inverted string solutions are characterized by the fact that the slope of the function $L(x)$ is constant and negative, $L(x)$ therefore crosses zero at some finite value of $x$, say $x=x_{I S}$. On the other hand, the Melvin branch evolves into the Kasner branch, a configuration for which the function $M(x)$ develops a zero at some finite value of $x=x_{K}$ while $L(x)$ becomes infinite for $x \rightarrow x_{K}$. More precisely, for $0<<x<x_{K}$ these solutions have the behaviour 
$M \propto\left(x_{K}-x\right)^{2 / 5}, L \propto\left(x_{K}-x\right)^{-3 / 5}$. The transition between Melvin solutions $($ for $\gamma<2)$ and Kasner solutions (for $\gamma>2$ ) is illustrated in Fig. 2 .

For negative $\Lambda$ and $\gamma<2$, the string and Melvin solutions are still present and merge into a single solution at some critical value of $\Lambda$. For $\Lambda<0$ and $\gamma>2$, we have the zero $x_{I S}$ of the inverted string solution increasing with decreasing $\Lambda$. It reaches infinity for the exponentially decreasing solution, the so-called "warped" solution that localises gravity on

the brane [16, 17]. If the cosmological constant is further decreased the solution becomes of Kasner-type.

\section{Positive bulk cosmological constant $(\Lambda>0)$}

Up to now static branes have only been discussed in an asymptotically flat or Anti-de Sitter bulk. Here, we also consider the case of static branes in a de Sitter bulk. First, we examine the evolution of the string and Melvin solutions for $\Lambda>0$. For all solution constructed with $\Lambda>0$, we were able to recover the behaviour (22) asymptotically.

This evolution is illustrated in Fig 3 and Fig 4 for $\gamma=1.6$ respectively for the string and Melvin solutions. Here, we show the metric functions $M, L$ for $\Lambda=0$ and $\Lambda= \pm 0.005$. For $\Lambda>0$ we find a solution with the metric function $M(x)$ possessing a zero at some finite $x$, say $x=x_{1}$. At the same time $L\left(x \rightarrow x_{1}\right) \rightarrow+\infty$. These solutions tend to the string solutions in the limit $\Lambda \rightarrow 0$. Following the convention used in the $\Lambda=0$-case, we refer to these solutions as of "Kasner"-type.

The second type of solutions that we find has metric functions behaving for $0<x_{2}<x_{3}$ as

$$
L\left(x_{2}\right)=0, M^{\prime}\left(x_{2}\right)=0, M\left(x_{3}\right)=0, \lim _{x \rightarrow x_{3}} L(x)=-\infty .
$$

These tend to the Melvin solutions in the limit $\Lambda \rightarrow 0$ and we will refer to them as of "inverted string"-type.

For fixed $\Lambda>0$ and increasing $\gamma$ we find that both Kasner and inverted string solutions exist for all values of $\gamma$. This is demonstrated in Fig 5 where the values of the parameters $C_{1}, C_{2}, x_{0}$ (defined in Eq.(22) $)$ are plotted as functions of $\gamma$. 


\section{B. Inflating branes $\kappa>0$}

Here, we discuss inflating branes $(\kappa>0)$. Again, we fix $\alpha=2.0$. Since observational evidence points to a positive cosmological constant in our universe (which is represented by the inflating 3 -brane) we restrict to $\kappa>0$ here.

The pattern of solutions can largely be characterized by the integration constant $C$ appearing in (24).

\section{Zero bulk cosmological constant}

First, we have constructed solutions corresponding to deformations of the string solutions residing in the extra dimensions. We present the profiles in Fig. [6] for $\kappa=0.003$ and for comparison for $\kappa=0$. Obviously, the presence of an inflating brane $(\kappa>0)$ changes the asymptotic behaviour of $M, L$ drastically in comparison to a Minkowski brane. $M(x)$ now behaves lineary far from the core of the string, while $L(x)$ tends to a constant. The solutions approach asymptotically (24). The space-time is thus cigar-like.

This can be explained as follows : In the case $\alpha=2$ the equations are self dual, in particular the equation determining the function $M$ on the string branch is $M M^{\prime \prime}+(3 / 2)\left(M^{\prime}\right)^{2}-$ $\kappa / 2=0$ (the combination of the energy momentum tensor vanishes identically for $\alpha=2$ and for the string like solution). The value of $C$ compatible with the boundary condition turns out to be $C=-\kappa / 4$, since we are interested is small values of $\kappa$, the integral (24) can be reasonably approximated by (25), in complete agreement with our numerical results.

The parameters $L_{0}$ and $C$ appearing in (24) can be determined numerically. It turns out that for the cigar-like solutions we always have $C<0$, while $L_{0}$ is positive. For a fixed value of $\kappa$ and varying $\gamma$, we find that $C \rightarrow 0$ for a critical, $\kappa$-dependent value of $\gamma, \gamma_{c r}(\kappa)$. At the same time $L_{0} \rightarrow 0$ for $\gamma \rightarrow \gamma_{c r}$. This is shown for three different values of $\kappa$ in Fig[7] and Fig 8 . In the limit $\gamma \rightarrow \gamma_{c r}$, the diameter of the cigar tends to zero and the cigar-like solutions cease to exist.

We have also studied solutions corresponding to deformations of the Melvin solutions. It turns out that the $\kappa=0$ Melvin solution is smoothly deformed for $\kappa>0$. $C$ is always positive and tends exponentially to zero as function of $\gamma$. For $\gamma>2$ we find that the solutions have a zero of the metric function $L$ at some $\gamma$ - and $\kappa$-dependent value of $x$. The solutions 
are thus of inverted string-type.

We present the pattern of solutions in the $\gamma$ - $\kappa$-plane in Fig 9

\section{Positive/negative bulk cosmological constant}

We have limited our analysis here to $\gamma \leq 1.8$. For $\gamma>1.8$, the numerical analysis becomes very unreliable, that is why we don't report our results here.

Fixing $\gamma$, e.g. to $\gamma=1.8$, the inverted string solutions (available for $\Lambda>0, \kappa=0$ ) gets smoothly deformed for $\kappa>0$. The constant $C$ is positive for all solutions. For fixed $\kappa$ and $\Lambda \rightarrow 0$ the Melvin solution is approached.

The Kasner solutions (for $\Lambda>0$ ) get deformed and the functions $M, L$ become periodic in $x$ asymptotically. These are deformations of the periodic solution (26) with $C \neq 0$. This is illustrated in Fig 10, The value of $C$ for these solutions is negative. The function $M$ oscillates around a mean value given by $M_{m v}=\left(\frac{-3 C}{2 \Lambda}\right)^{1 / 5}$ and stays strictly positive, the solution is therefore regular. The period of the solution depends weakly on $\kappa$. In the limit, $\Lambda \rightarrow 0$ the periodic solutions tend to the cigar-like solutions. As mentioned above, we notice that the values of the constants $\kappa$ and $\Lambda$ can be chosen in such a way that $2 \kappa-\Lambda$ becomes arbitrarily small. This seems compatible with the domain of parameters leading to periodic solutions. The corresponding Planck mass determined through (19) can therefore be made arbitrarily large.

A sketch of the pattern of the solutions is proposed in Fig [11. The fact that we obtain periodic solutions constitutes a natural framework to define finite volume brane world models. With view to the discussion on the effective 4-dimensional action, we could imagine to put branes with "large" effective gravitational coupling at $x=x_{k}, k=1,2,3, \ldots$, where $x_{k}$ corresponds to the position of the maxima of $M(x)$. Correspondingly, branes with "small" effective gravitational coupling could be put at $x=\tilde{x}_{k}, k=1,2,3, .$. , where $\tilde{x}_{k}$ are the positions of the minima of $M(x)$. Though there is no "warping" in the sense of Randall and Sundrum [7, 8], nevertheless gravity looks stronger on the branes positioned at the maxima of $M(x)$ in contrast to gravity on the branes positioned at the minima. Note that at the positions of these branes, the metric function $L(x)$ vanishes, i.e. $L\left(x_{k}\right)=L\left(\tilde{x}_{k}\right)=0$. Thus the cylindrical geometry of the extra dimensional space shrinks to a point and the whole space-time geometry becomes effectively 5-dimensional. 


\section{SUMMARY AND DISCUSSION}

We have studied static as well as inflating brane solutions in a 6-dimensional brane world scenario with an abelian string residing in the two extra dimensions. The pattern of the solutions is very rich, leading to many different types of behaviours of the gravitating fields (depending on the values of $\gamma, \kappa$ and $\Lambda$ ) outside the core of te string. We find it convenient to summarize the possiblilites as follows

- String solutions (S) with $M \rightarrow 1, L \rightarrow \infty$ for $x \rightarrow \infty$,

- Melvin solutions (M) with $M \rightarrow \infty, L \rightarrow 0$ for $x \rightarrow \infty$,

- Inverted string solutions (IS) with $M \rightarrow 0, L \rightarrow-\infty$ at some finite value of $x$ $\left(L\left(x_{1}\right)=M^{\prime}\left(x_{1}\right)=0\right.$ for some intermediate value of $\left.x=x_{1}\right)$,

- Kasner solutions $(\mathrm{K})$ with $M \rightarrow 0, L \rightarrow \infty$ at some finite value of $x$,

- Cigar-type solutions (C) with $M / x \rightarrow M_{0}, L \rightarrow L_{0}$ for $x \rightarrow \infty$,

- Periodic solutions (Per) with $M, L$ periodic in the bulk coordinate $x$.

We point out again here that the different names used to characterize the solutions is an adaptation of the terminology used e.g. in [19] to the present context.

Evaluating the different curvature invariants of the metric we find that the Kasner and inverted string solutions possess a physical singularity at the value of $x$ at which the function $M$ vanishes. Note that this excludes the inverted string solutions for static branes in an asymptotically flat bulk, where $M$ possesses no zero. These solutions were shown to be regular [17]. All the other space-times are without physical singularities. If the symmetry breaking scale is below a critical below (given by the higher-dimensional Planck scale) corresponding to $\gamma<2$, the space-time containing a static brane is singularity-free for an asymptotically flat or Anti-de-Sitter bulk, however has singularities for an asymptotically de Sitter bulk (positive bulk cosmological constant). Inflating branes lead to a cigar-like universe for vanishing bulk cosmological constant at a symmetry breaking scale below a specific value that depends on the value of the brane cosmological constant. Next to these, also Melvin solutions exist, which again are singularity-free. 
If the symmetry breaking scale is above a critical value, the space-time is singular for static branes in an asymptotically flat bulk $(\Lambda=0)$. If we replace the static brane by an inflating brane, the space-time still possesses a singularity. Equally, the introduction of a positive cosmological constant doesn't remove the singularity of the space-time.

If both the bulk cosmological constant and the brane cosmological constant are nonvanishing, regular space-times are possible, namely periodic solutions with non-vanishing minima of the metric functions. These appear if in the space-time with $\Lambda>0$ (Kasner solutions) the static brane is replaced by an inflating brane. If one puts branes at the respective minima and maxima of these periodic solutions, a hierarchy between the effective 4-dimensional gravitational coupling is possible.

As a final remark let us relate our periodic solutions to recently investigated solutions in higher dimensional space-times, namely "Non-uniform black strings" (see e.g.

23]). Indeed, referring to Fig.1 of [23], the solution in the extra dimension could be continued periodically by gluing several copies of the solution. The structure in the extra dimension then becomes kind of periodic. The crucial difference, of course, is that in the present paper we don't have black holes extended into extra dimensions, but an inflating brane (the 3-brane) with two extra dimensions. However, again, we find a periodicity of the solution in the extra dimension, in our case in the $x$-direction. The periodic solutions presented here could thus be seen as "non-uniform De Sitter-vacuua".

\section{Acknowledgements}

YB is grateful to the Belgian FNRS for financial support.

[1] T. Kaluza, Sitzungsber. Preuss. Akad. Wiss. Berlin K1 (1921), 966; O. Klein, Z. Phys. 37 (1926), 895 .

[2] see e.g. J. Polchinski, String Theory, Cambridge University Press (1998).

[3] V. A. Rubakov and M. E. Shaposhnikov, Phys. Lett. B 125 (1983), 136; 125B (1983), 139.

[4] G. Dvali and M. Shifman, Phys. Lett. B 396 (1997), 64; 407 (1997), 452.

[5] I. Antoniadis, Phys. Lett. B 246 (1990), 377.

[6] N. Arkani-Hamed, S. Dimopoulos and G. Davli, Phys. Lett. B429 (1998), 263; I. Antoniadis, 
N. Arkani-Hamed, S. Dimopoulos and G. Dvali, Phys. Lett. B 436 (1998), 257.

[7] L. Randall and R. Sundrum, Phys. Rev. Lett. 83 (1999), 3370.

[8] L. Randall and R. Sundrum, Phys. Rev. Lett. 83 (1999), 4690.

[9] K. Akama, Pregeometry in Lecture Notes in Physics, 176, Gauge Theory and Gravitation, Proceedings, Nara, 1982, edited by K. Kikkawa, N. Nakanishi and H. Nariai, 267-271 (SpringerVerlag,1983), hep-th/0001113

[10] T. Gherghetta, E. Roessl and M. E. Shaposhnikov, Phys. Lett. B 491 (2000), 353.

[11] O. DeWolfe, D. Z. Freedman, S. S. Gubser and A. Karch, Phys. Rev. D 62 (2000), 046008.

[12] T. Gherghetta and M. E. Shaposhnikov, Phys. Rev. Lett. 85 (2000), 240.

[13] M. Giovannini, H. B. Meyer and M. E. Shaposhnikov, Nucl. Phys. B 691 (2001), 615.

[14] E. Roessl and M. E. Shaposhnikov, Phys. Rev. D 66 (2002), 084008.

[15] I. Cho and A. Vilenkin, Phy. Rev. D 68025013 (2003); I. Cho and A. Vilenkin, Phy. Rev. D 69045005 (2003).

[16] Y. Brihaye and B. Hartmann, Nucl. Phys. B 691 (2004), 7.

[17] Y. Brihaye and B. Hartmann, Int. J. Mod. Phys. A 11 (2005), 2265.

[18] H. B. Nielsen and P. Olesen, Nucl. Phys. B 61 (1973), 45.

[19] M. Christensen, A. L. Larsen and Y. Verbin, Phys. Rev. D 60 (1999), 125012; Y. Brihaye and M. Lubo, Phys. Rev. D 62 (2000), 085004.

[20] I. Olosagasti and A. Vilenkin, Phys. Rev. D 62 (2000), 044014.

[21] E. R. Bezerra de Mello, Y. Brihaye and B. Hartmann, Phys. Rev. D 67 (2003), 124008.

[22] B. Linet, J. Math. Phys. 27 (1986), 1817.

[23] T. Wiseman, Class. Quant. Gravity 20 (2003), 1177. 


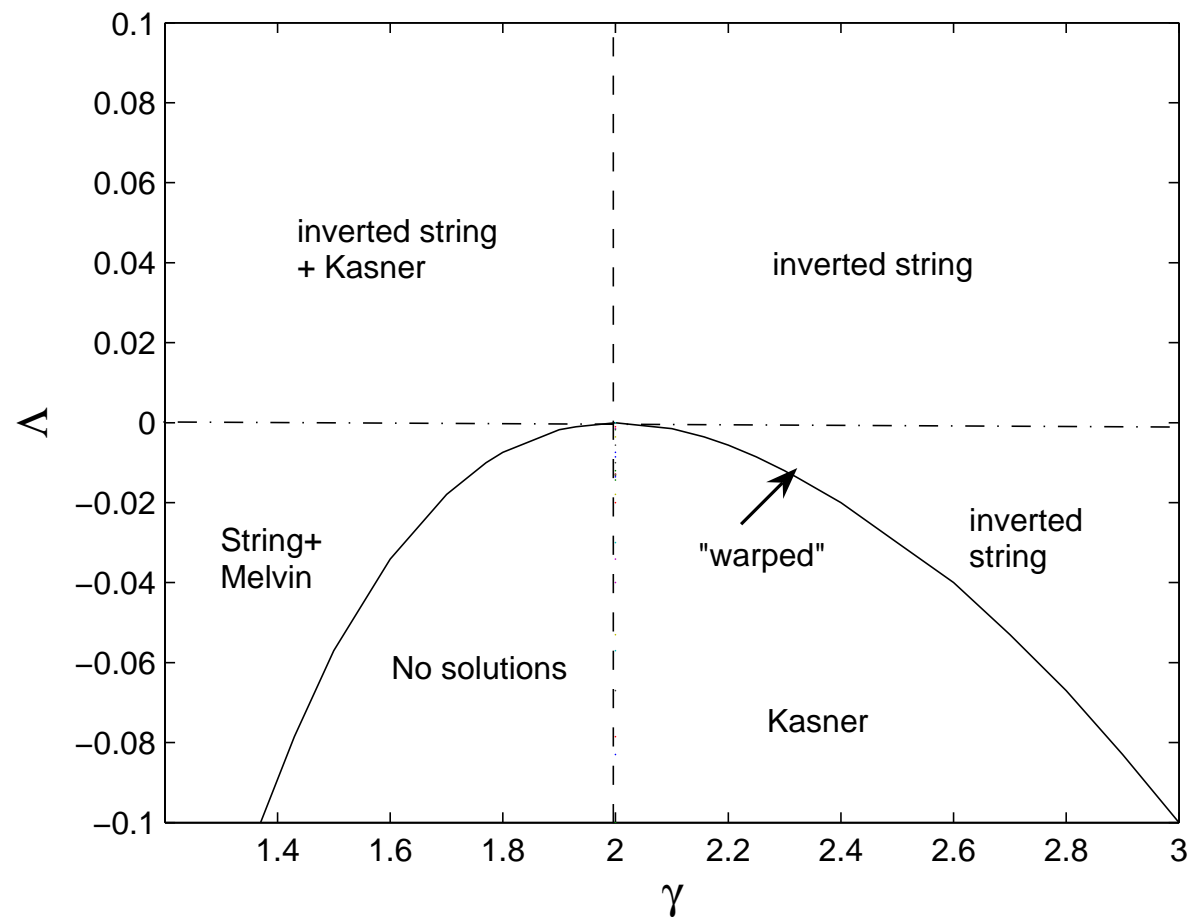

FIG. 1: The pattern of brane world solutions in the $\Lambda-\gamma$ plane for $\alpha=2$. 


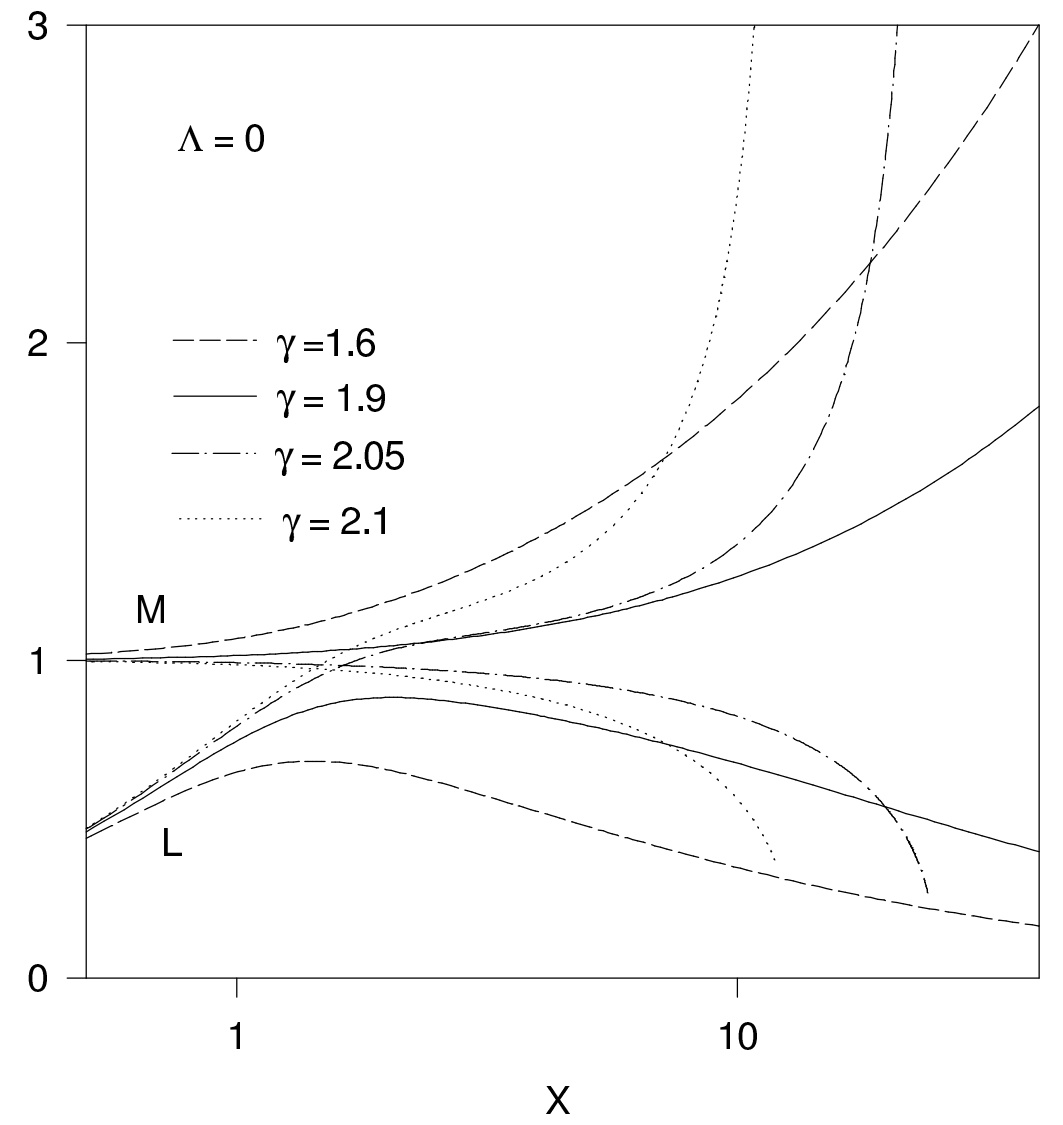

FIG. 2: The pattern of the transision from the Melvin solution $(\gamma<2)$ to the Kasner solution $(\gamma>2)$ for $\Lambda=0$ if given for the metric functions $M$ and $L$. 


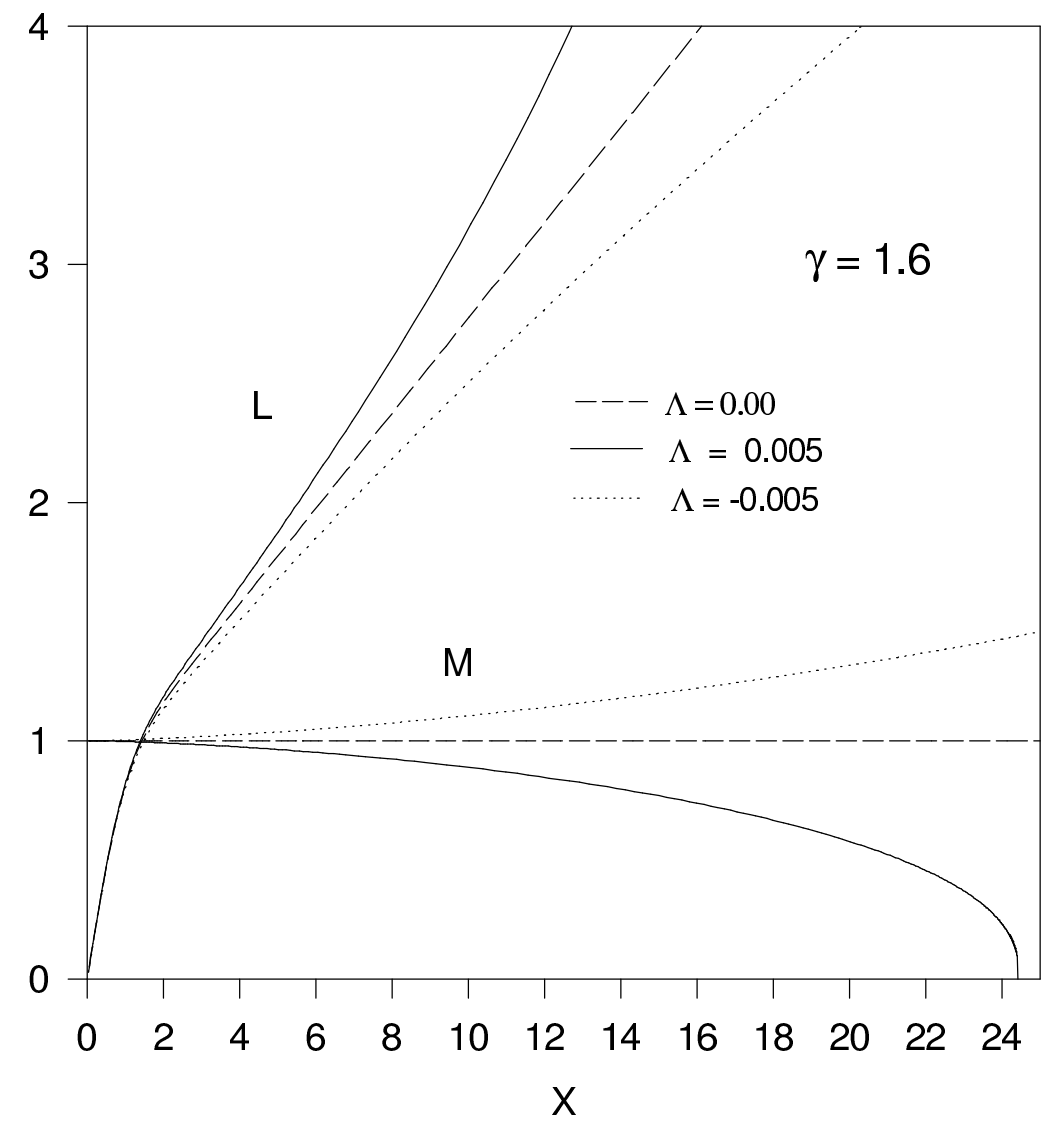

FIG. 3: The profiles of the metric functions $M, L$ of the string solution (for $\Lambda=0, \Lambda=-0.005$ ), respectively of the Kasner solution (for $\Lambda=0.005$ ) for $\gamma=1.6$. 


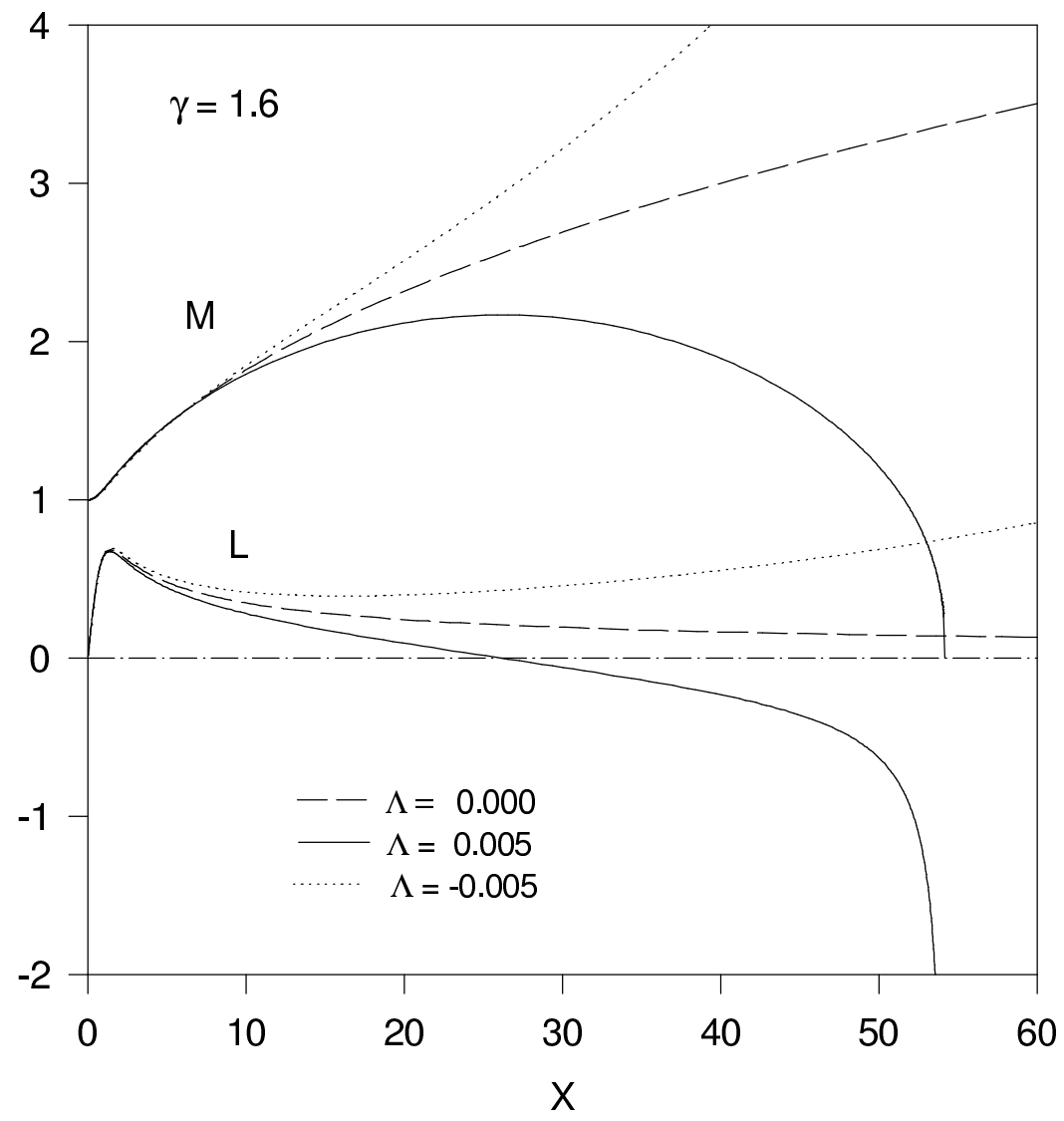

FIG. 4: The profiles of the metric functions $M, L$ of the Melvin solution (for $\Lambda=0, \Lambda=-0.005$ ), respectively of the inverted string solution (for $\Lambda=0.005$ ) $\gamma=1.6$. 


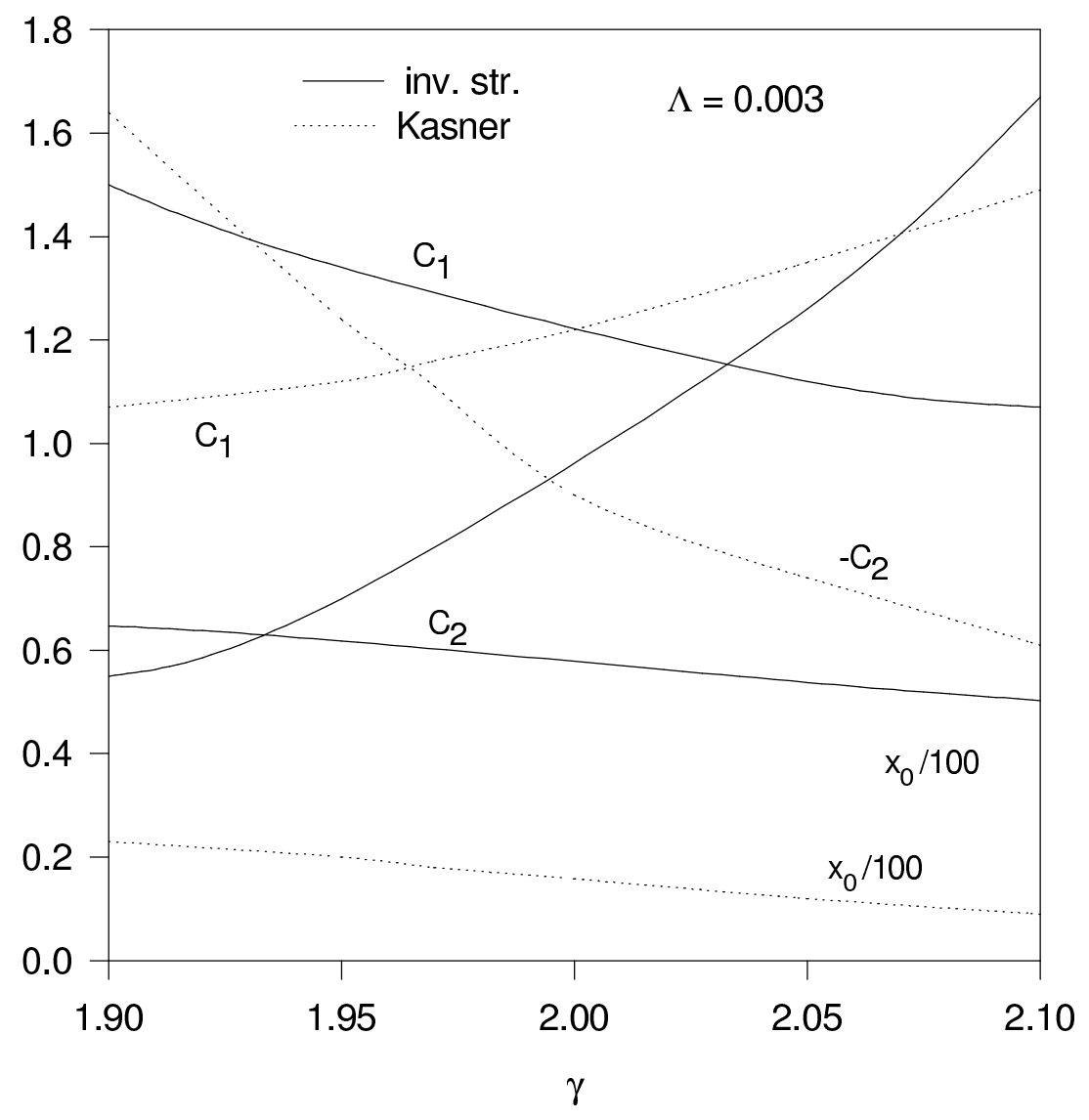

FIG. 5: The values of the parameters $C_{1}, C_{2}, x_{0}$ defined in the text for the inverted string and Kasner-like solutions as functions of $\gamma$ for $\Lambda=0.003$ 


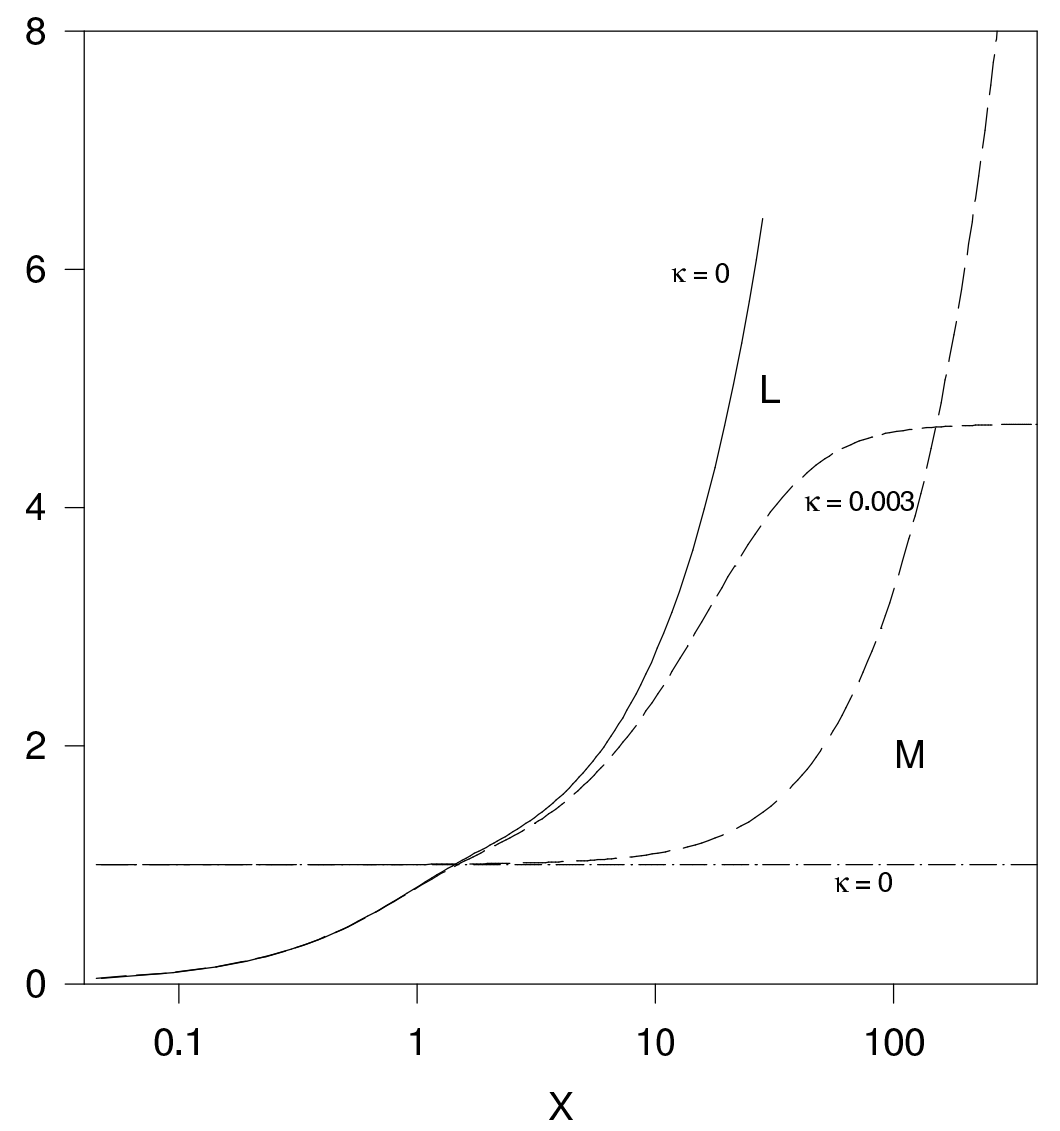

FIG. 6: The profiles of the metric functions $M, L$ for $\Lambda=0$ and for $\kappa=0$ and $\kappa=0.003$, respectively. 


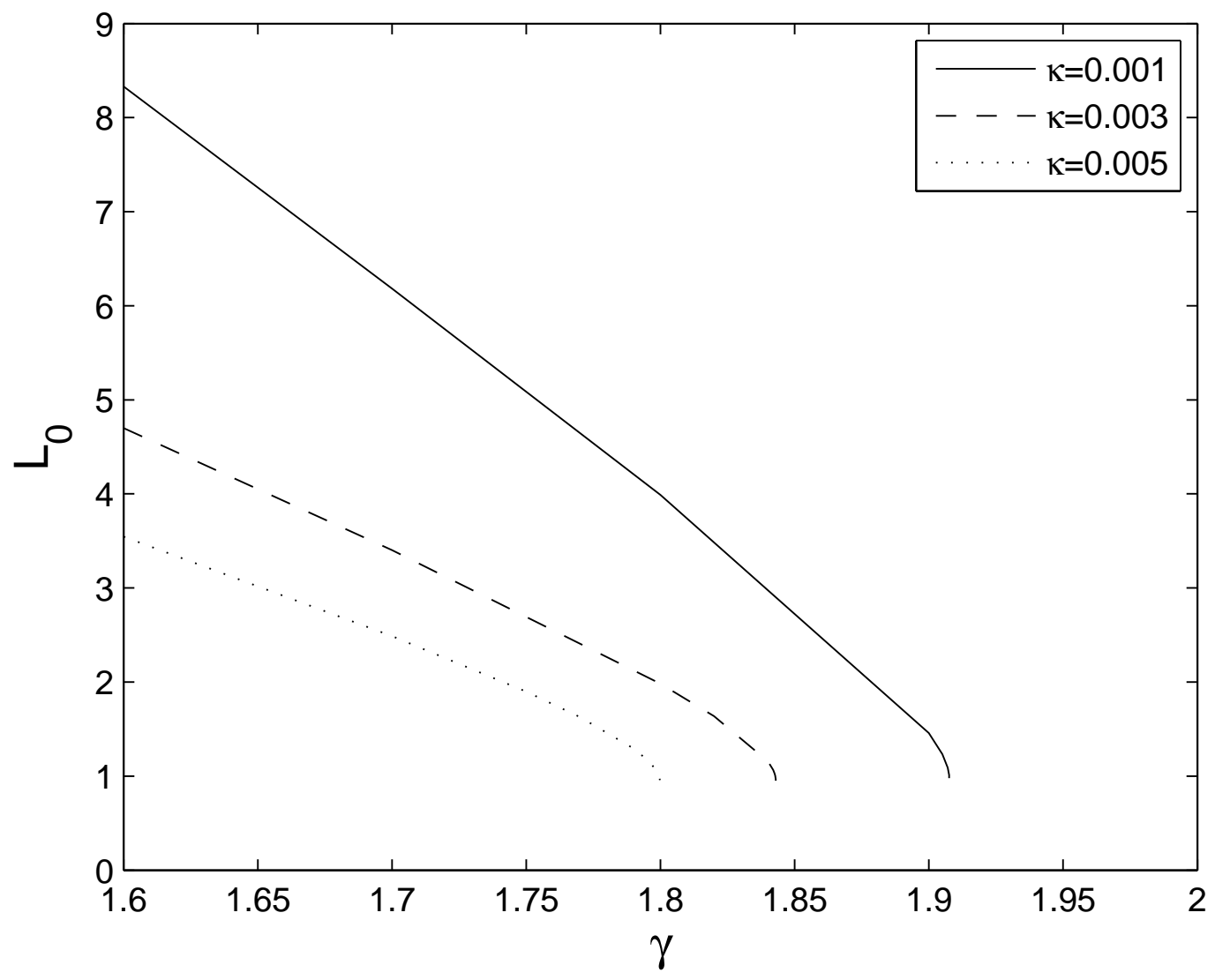

FIG. 7: The parameter $L_{0}$ of the cigar-like solution as function of $\gamma$ for different values of $\kappa$ and $\Lambda=0$. 


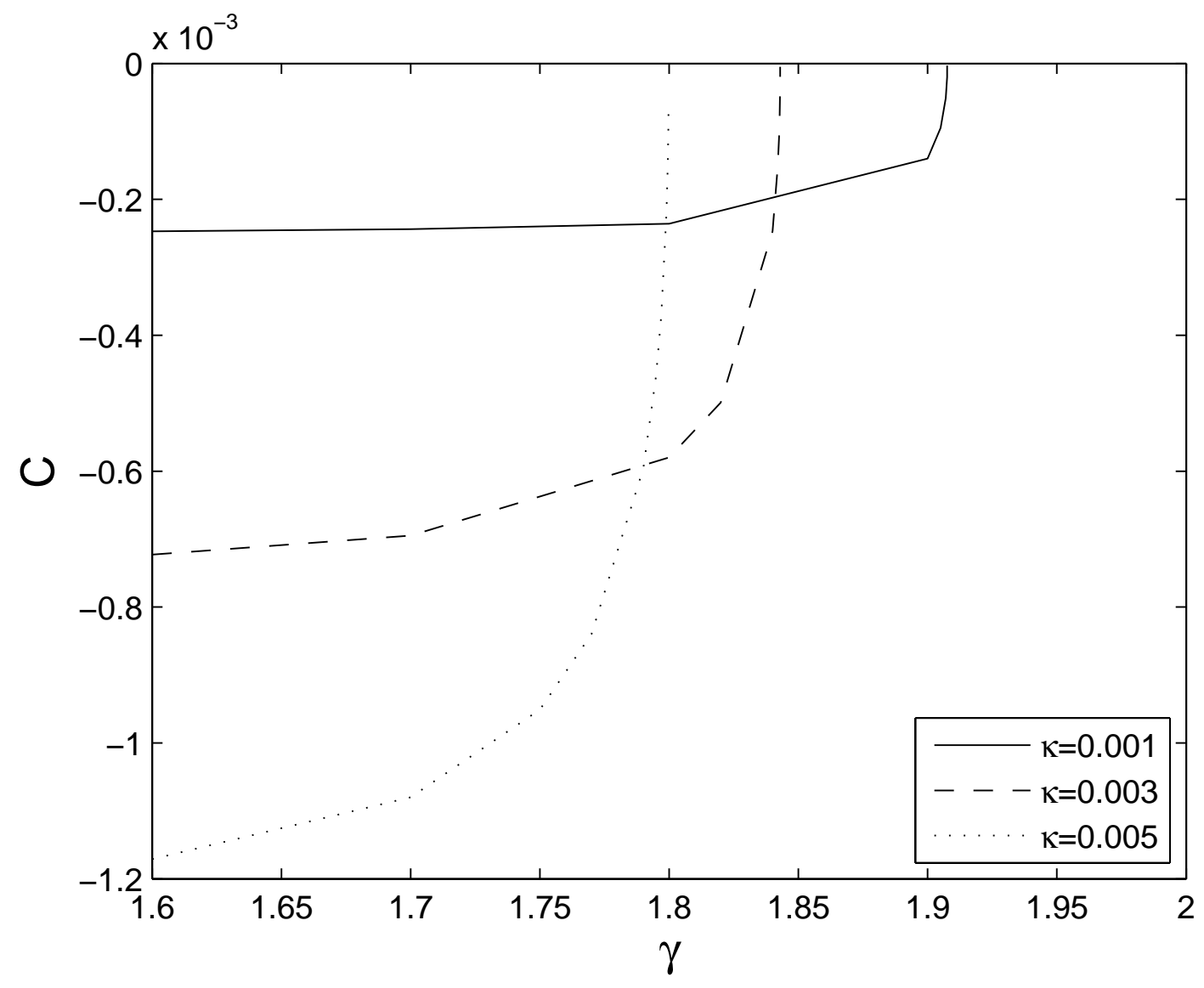

FIG. 8: The parameter $C$ of the cigar-like solution as function of $\gamma$ for different values of $\kappa$ and $\Lambda=0$. 


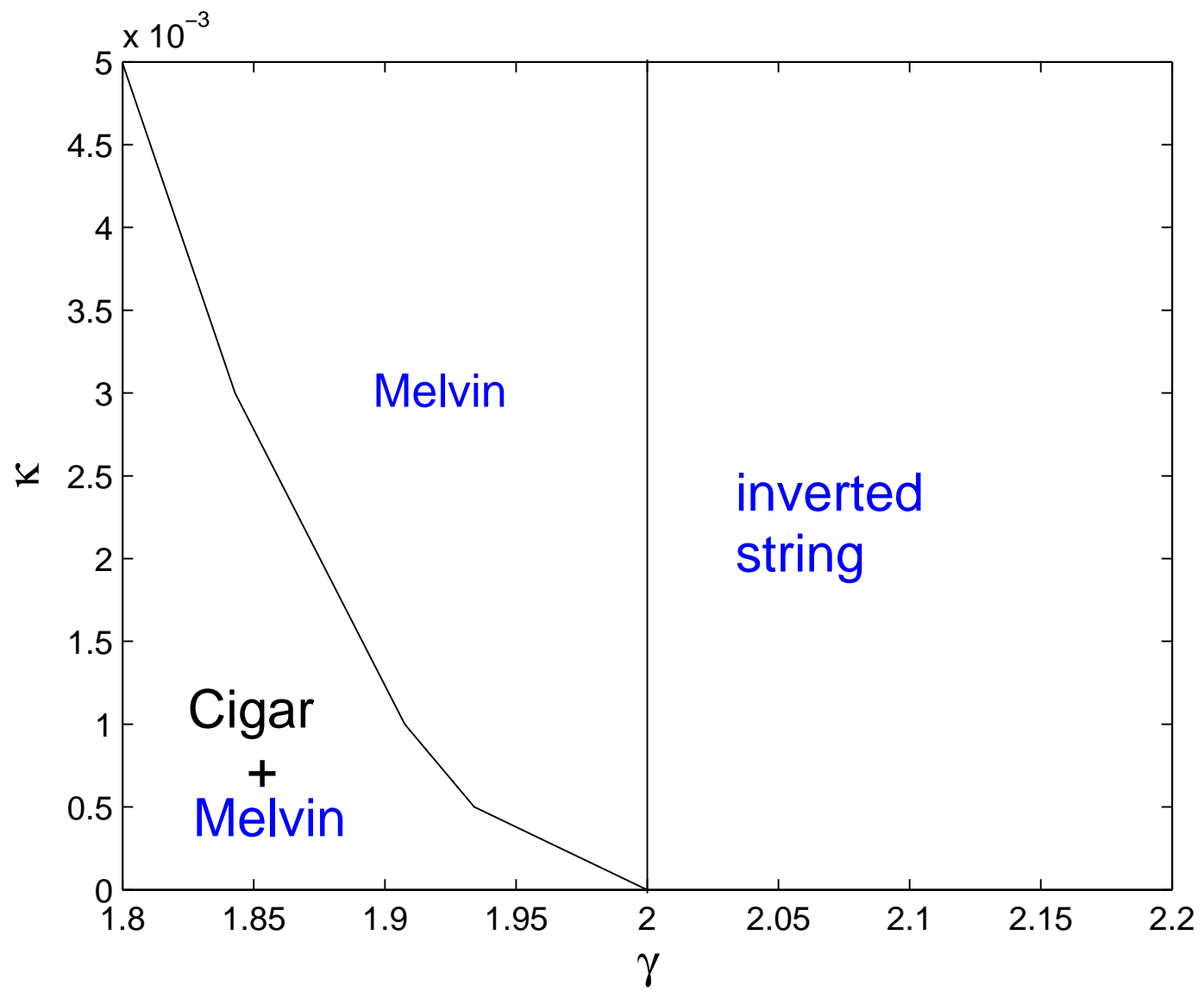

FIG. 9: The pattern of inflating brane solutions in the $\gamma$ - $\kappa$-plane. 


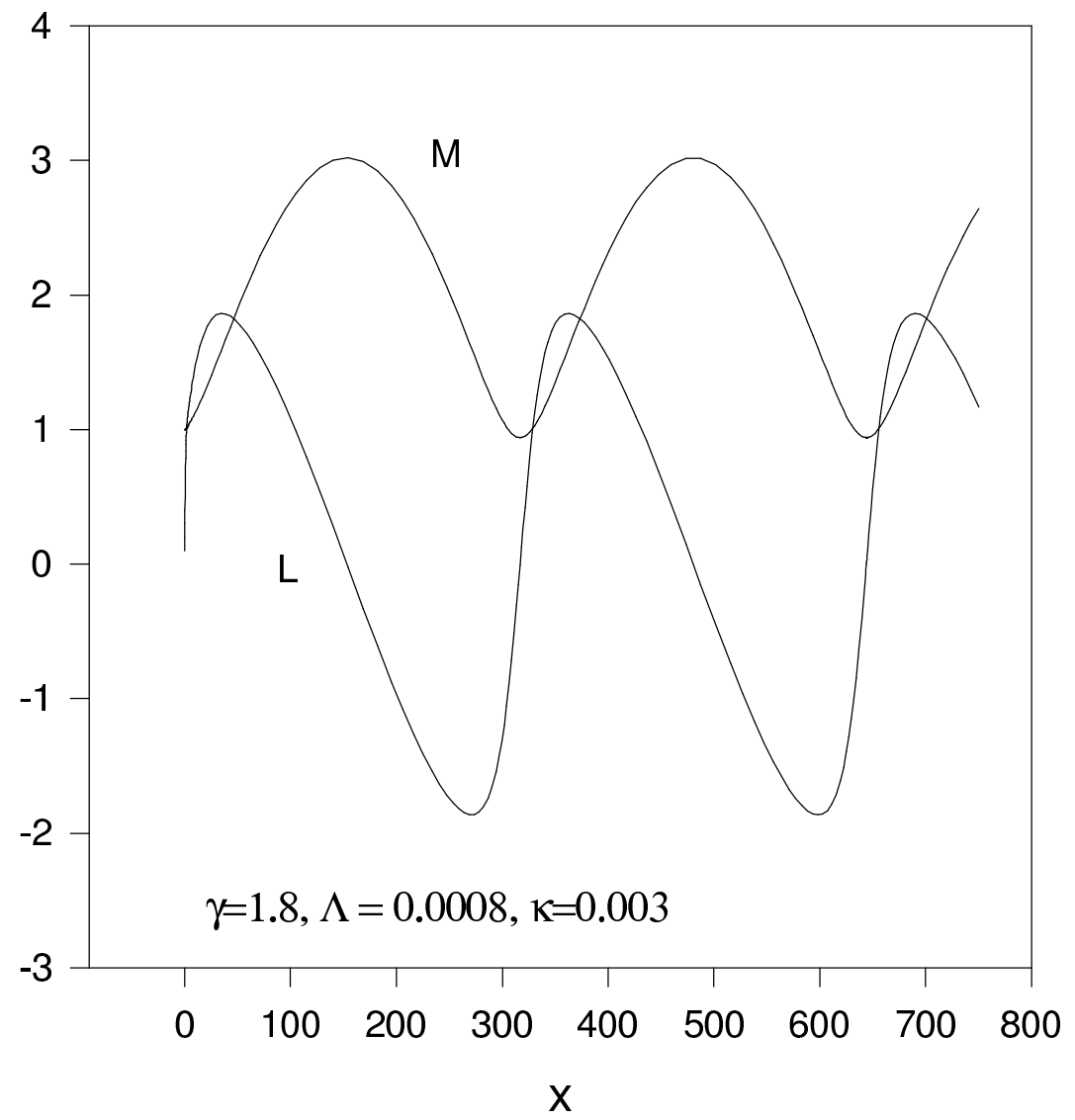

FIG. 10: The profiles of the metric functions $M, L$ for $\Lambda=0.0008$ and for $\kappa=0.003$ and $\gamma=1.8$. 


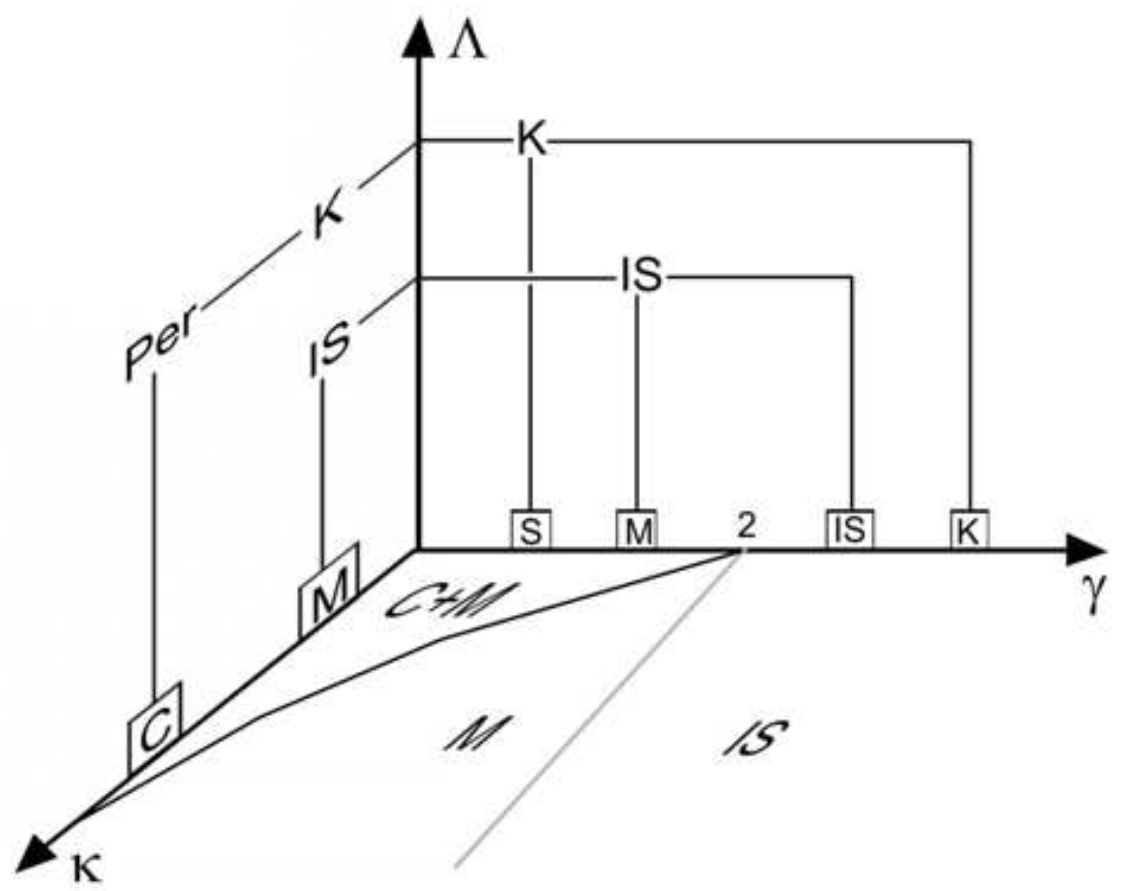

FIG. 11: The pattern of brane solutions in the $\kappa-\gamma$ - $\Lambda$-domain. The origin of the coordinate system here corresponds to $\kappa=0, \gamma=1.8, \Lambda=0$. M, S, IS, K, Per, C denote Melvin, string, inverted string, Kasner, periodic and cigar-type solutions, respectively. 